EOMmun Communication et organisation

Or

Repenser la communication dans les organisations

publiques

\title{
Entretien avec Pierre Zemor
}

\section{(2) OpenEdition}

\section{Journals}

Édition électronique

URL : http://journals.openedition.org/communicationorganisation/867

DOI : 10.4000/communicationorganisation. 867

ISSN : $1775-3546$

Éditeur

Presses universitaires de Bordeaux

Édition imprimée

Date de publication : 1 juin 2009

ISSN : 1168-5549

Référence électronique

«Entretien avec Pierre Zemor », Communication et organisation [En ligne], 35 | 2009, mis en ligne le 10

mars 2011, consulté le 14 septembre 2020. URL : http://journals.openedition.org/

communicationorganisation/867 


\section{Entretien avec Pierre Zemor}

Pierre Zémor est conseiller d'Etat honoraire, membre de la Commission des sondages, président fondateur de l'association "Communication publique" et de la fédération européenne FEACP. Ancien consultant en management et en communication politique, élu local et régional, il a enseigné à HEC, à Sciences-Po, et à l'ENA.

\section{Pour vous, quelle est la spécificité irréductible de la communication publique?}

L'une des définitions du client, c'est qu'il est roi et qu'il a le choix de son service. Or chez l'usager citoyen, il y a un paradoxe quelque part. L'utilisateur du service public est roi au sens où il partage le pouvoir, il est la source de sa légitimité. Pour autant, il est usager sans alternative de choix, car l'offre de service est d'intérêt général, et la construction de cet intérêt dans une démocratie représentative est le fruit des institutions. Le citoyen, usagé du service public, contribuable, qui se sent partisan de la décision stratégique et qui est usager d'un service offert, vit donc au quotidien ce paradoxe. Donc, quand dans les sondages, on interroge les gens, il y a vraiment plus que dans le passé, l'envie d'avoir un état ayant un rôle protecteur et régulateur. Et dans le même temps, les gens disent qu'avant tout ils veulent être considérés comme citoyens, particulièrement dans la communication des institutions publiques. Mais il faut faire attention aux simplifications. C'est une illusion de penser que la majorité des personnes veulent être dans un système de codécision. La plupart des citoyens disent ne pas vouloir ou pouvoir décider et estiment avoir des représentants pour ça. Mais les citoyens veulent être pris en compte dans un processus de décision transparent. C'est la culture de la concertation et du débat public qui innerve toutes les pratiques de la communication publique.

Les gens qui disent « la communication, c'est de la pub, il suffit de prendre des techniques et on fait du marketing » se trompent, ce n'est pas pertinent pour une institution publique. Le marketing n'est pas de la communication. J'ai enseigné la stratégie à HEC, donc je saisis assez bien la force du marketing dans un environnement concurrentiel pour gagner des parts de marché. Si l'usager du service public doit être considéré comme un client, c'est un minimum pas une fin en soi. Il est au minimum un client. Cette approche client centrée a été une avancée importante, à partir des années 70/80, dans la communication de l'administration et des services publics, qui avait pris un retard certain. Il a fallu descendre le service public de son piédestal dans les 


\section{Entretiens}

années 1980. Les registres de la communication publique ne sont pas seulement de promouvoir les services offerts au public. Il s'agit d'informer le citoyen : c'est une information qui est due au citoyen. Nous sommes au cœur de la problématique de la mise à disposition des données publiques.

La charte déontologique de l'association de la communication publique ${ }^{191}$ prône la responsabilité et le devoir envers les citoyens. Dès qu'un responsable de communication parle, il dépend de sa hiérarchie et dépend aussi de son devoir à l'égard des citoyens, il a cette double responsabilité qui forge une véritable déontologie professionnelle, qui peut aboutir à refuser une certaine communication dans l'institution.

Vous avez été au cour de l'émergence de la communication publique à une période où il fallait faire un énorme travail de définition de cette communication et l'extraire du marketing publicitaire et des publicitaires eux-mêmes. Comment cette nécessité vous est-elle venue?

Il faut extraire la communication publique de la publicité en s'appuyant sur sa légitimité et l'identité de son annonceur... Ce sont les deux frontières sur lesquelles il existe des batailles pour affirmer la communicabilité d'un message public. On doit rendre des comptes à des citoyens, envers qui on a un devoir, ce qui n'a strictement rien à voir avec de la communication mercantile. Mais quand on veut la mettre en évidence, on a une tentation de réduction de la communication publique à la communication concurrentielle. D'ailleurs, la présence forte des publicitaires et des sciences du marketing dans le métier nous interroge en permanence sur ses frontières. Mais, les mass médias ont aussi une fâcheuse tendance à tout mettre dans le même sac : promotion égale manipulation, etc. Les frontières entre la communication concurrentielle et la communication publique sont parfois trop pervasives, les tentations sont nombreuses. Les médias ont étendu la critique du service public à la communication publique. Affirmer sa légitimité nécessite une mise en évidence. La communication gouvernementale est à cheval sur cette frontière. Elle se veut institutionnelle mais aussi la valorisation des compétences d'une équipe gouvernementale. La communication politique est, quant à elle, clairement une communication promotionnelle concurrentielle. Car la classe politique n'est pas arrivée à bâtir une identité propre à l'élu en gouvernement. Du fait de

${ }^{191}$ http://www.communication-publique.fr/charte.html 
l'accélération des agendas électoraux, l'homme politique est en campagne permanente. Or la conquête du pouvoir est en situation concurrentielle. C'est cela qui apparaît. Les élus qui font de la communication publique sur le long terme apparaissent au sein même de leur équipe comme des naïfs ou des utopistes. Le moindre échec électoral et la conversion au tout marketing n'est pas loin.

Ce problème d'informer tout en ayant une relation de qualité et pérenne avec le citoyen. Il y a des moments où c'est contradictoire avec le fait de promouvoir l'image de l'institution publique. Nous avons alors une contradiction entre le devoir d'informer et la relation de valorisation de l'action publique. Cela est en quelque sorte le point de contradiction dans l'institution administrative qui est aussi une instance politique. S'il faut une communication promotionnelle, en réalité, on s'aperçoit qu'il faut faire une communication attendue par le public c'est-à-dire aborder la notion didactique (qu'est ce que c'est que telle et telle chose). Donc il y a ce problème entre la notion d'informer et la relation de qualité avec les citoyens. C'est un problème entre faire valoir et faire savoir. Il y a aussi une très forte demande chez le public pour une information didactique. Mais il y a aussi un devoir de proximité et d'écoute qu'il est nécessaire de ne pas oublier. On retrouve cette ambigüité sur l'information gouvernementale qui aide et soutient les campagnes.

En période de crise, les entreprises ont baissé leurs budgets parce qu'elles trouvent qu'on a fait trop de pub... il y a un ralentissement. Et, en parallèle, il y a une reconnaissance de la communication publique car elle est là comme une protection, comme une assurance de l'institution. On revient à la définition de l'État qui est régulation, protection et anticipation. Il y a beaucoup d'avancées. Depuis plus de 20 ans, j'ai des contacts avec l'ENA qui souhaitait faire du média planning, faire venir des agences, pour autant nous en sommes à l'embryon d'une véritable formation en communication publique. Quand j'ai fait mon cours à Science Po sur la communication publique, j'ai dit « la communication est là pour s'intégrer à l'action ». Par conséquent, la communication est comprise dans le processus. Si la communication s'en dissocie, on peut craindre le pire. Avant la communication était en plus ; maintenant, c'est une communication vécue comme nécessairement très intégrée. Mais il y a une école, très influencée par la communication politique, qui dit que la communication est en elle même une action. On peut considérer que certains éléments de communication sont des actions, mais ce n'est pas le cas de tous. 


\section{Entretiens}

\section{Il y aurait une troisième définition entre communication politique et institutionnelle qui serait proche d'une sorte de communication gouvernementale.}

La communication gouvernementale est complètement à cheval sur cette définition. Car, elle est normalement institutionnelle. C'est à dire, quand les lois sont votées on ne peut rien changer. Mais les dérives actuelles, avec les participations des gens d'un SIG orienté pub, de plus en plus omniprésent dans le cabinet du Premier ministre, la nomination d'un délégué interministériel à un rang gouvernemental, fait qu'il n'y a plus vraiment de frontières entre action publique et marketing. Dans l'absolu, c'est vrai, toute communication institutionnelle a la légitimité du politique. Mais dans la pratique, au moins $80 \%$ des activités de communication d'une institution publique seraient les mêmes quelle que soit la majorité ou les patrons. C'est une communication sur le long terme, c'est plus technique et juridique.

Aujourd'hui, vous avez un très fort progrès de la communication institutionnelle territoriale. Cette pratique est très développée entre la communication politique et institutionnelle depuis la décentralisation. $\mathrm{Si}$ les villes se vivent aujourd'hui en concurrence pour les investissements ou encore le tourisme, la décentralisation leur a donné comme travail de rendre l'état de droit familier. C'est-à-dire d'expliquer, de débattre ... La communication territoriale fait de nets progrès vers la communication publique en délaissant progressivement le tout marketing. La question du budget de communication rencontre encore de nombreux freins en collectivité du fait d'une idéologie ancienne qui en fait une dépense superfétatoire. Ce qui fait que tous les services n'ont pas forcément un budget de communication. Le problème de la communication territoriale est aussi un défi. Elle se trouve dans un système d'émetteurs cacophoniques. Ce n'est pas leur faute mais les collectivités, et les services déconcentrés, ne se rendent pas compte qu'il ne faut pas multiplier les sources d'information car le citoyen n'y comprend plus rien. Le résultat doit être collectif, thématique et en même temps, l'identité doit être individuelle. On doit donc éviter «le heurt des ego sur les logos » en quelque sorte.

Ce qui explique votre attachement au débat public. Le débat public est l'instance qui n'est pas réductible au pur marketing, du moins qui ne devrait pas l'être.

Le débat public est, en fin de compte, au cœur de la spécificité de la communication. Il y a des politiques qui commandent des débats publics. La communication était, dans un temps, réduite à 
l'information au sens noble. Puis, sont arrivés les publicitaires qui ont apporté la réclame et la promotion. Alors, on s'aperçoit que l'aspect dialogue est mis de côté. La présidentielle a remis à l'ordre du jour le dialogue, la participation à la communication publique. Il y a des élus qui ont compris que c'était très intéressant de faire de la communication publique authentique autour de la concertation. Mais, c'est vrai que la pression est très forte. «La communication est une forme de l'action publique » voilà notre débat actuel. Maintenant, on ne peut plus faire l'économie de ne pas intégrer la communication. Or l'écoute est la clé de toute communication réussie. Le débat public est au cœur de la spécificité de la communication. Il existe des temporalités différentes dans la communication: les stratégies de politiques publiques se mesurent sur deux décennies; la communication portant sur le fonctionnement du service public, cinq ans, les mandats politiques, un ou deux ans et, le temps des médias...deux ou trois jours.

L'espace public 2.0 veut dire le retour de l'usager, le citoyen ordinaire avec des médias comme internet dans le débat public de la communication.

La force des médias avec cette immédiateté (c'est-à-dire étymologiquement l'absence de médiation), est une séduction par un renoncement à la valeur ajoutée du jugement. Il y a une concurrence de l'immédiateté avec n'importe qui aujourd'hui. Avec internet, les choses sont devenues beaucoup plus complexes à penser. J'ai quelques réserves à répondre à une question qui est elle-même contaminée par une tendance forte dans l'adoption des usages. Car, le mot communication est souvent réduit. Le débat public n'a pas la même aura. Indubitablement, il y a intervention de sources qui existaient et qui n'avaient pas de possibilité d'être très vite présentes sur l'ensemble de la planète. Alors on appelle ça du web 2.0. Faut-il répondre à cette interrogation à la mode par une réponse structurelle qui engage la place de l'État dans la nation? Est ce que l'administration doit être 2.0 ?

A priori, mais avec réserve, je dirais non. Il y a pour l'administration publique un défi. On est à vie utilisateur du service public donc la qualité de la relation à avoir est importante, d'autant qu'elle porte sur la durée. La qualité de la relation et le contenu de l'information doivent être assurés avec prudence : une bonne communication est une information qui permet de faire valoir les messages dans un échange. C'est plutôt ça les objectifs. Et donc, la communication publique aun devoir de garantie des contenus. Elle repose sur une qualité et un 


\section{Entretiens}

contenu de l'information dans une relation délicate. Un contenu riche est le devoir d'une administration publique.

Il ne s'agit pas de raconter tout et n'importe quoi venant de sources non-investies légitimement en moins de 140 caractères par SMS. Quand les citoyens vont s'apercevoir qu'on leur raconte n'importe quoi, ils vont se retourner sur des valeurs sûres. Les médias n'ont pas de mémoires, alors que le service public doit être le garant de cette mémoire. De la même manière que les journalistes doivent refonder leurs travaux sur des bases classiques, on va avoir un retour aux valeurs de l'information validée. Une bonne communication a une mémoire mais les médias n'en ont pas. Il faut revenir à la mémoire, à la profondeur historique à la construction du temps par la communication publique. Le media, 2.0 ou pas, n'est pas en cause, c'est bien le sens de la stratégie de communication publique qu'il faut évaluer au regard de l'espace public.

Pourtant, le web 2.0, ce n'est pas seulement une mode, ça peut énormément perdurer. Mais ces phénomènes demandent un temps de décantation et de gestation qu'on n'a pas achevé. Laissons faire les innovateurs, réfléchissons, institutionnalisons les bonnes pratiques. Une institution publique peut avoir son propre site mais être très prudente sur le dialogue instantané sur internet. Par exemple dans le débat public, internet a pour fonction :

- De mettre l'information à la disposition des gens. Mais aussi de permettre l'accès à l'information à tous. Internet est une vitrine et un outil de diffusion.

- De pédagogie. Comprendre à la fois les contenus et processus de diffusion. Les gens interrogent beaucoup un débat. Vous avez là un rôle d'information interactive. Le rôle des pouvoirs publics est de trouver les gens qui sont capables de répondre des choses acquises et avérées.

- De forum : ouvert à tous et ma recommandation aux services publics est d'observer et ne pas s'en mêler. Il ne faut pas rentrer dans la discussion. On n'entre pas dans le débat si vous n'avez pas la force de répondre à tous, de vous y plonger pleinement.

Internet est un formidable outil pour permettre la diffusion et le débat politique mais attention à la limite de l'outil sur le dialogue et la progression des idées. D'ailleurs OBAMA l'a très bien compris, car son système n'était pas un dialogue sur le fond, mais il mise sur une mobilisation des gens qui voulaient parler sur le fond. On doit se méfier de ne pas tout mettre dans le dernier média mais s'attacher à 
choisir le bon média en fonction de la stratégie de communication même si internet devient chaque jour incontournable. Car tous les médias vont continuer à exister. C'est justement le rôle difficile des communicateurs publics qui est de trouver quel média utiliser pour quelle situation. Il y a une grande satisfaction des citoyens à s'informer. Et selon la culture des pays certains estiment que convaincre un échantillon de la population est suffisant. En France non, on a une culture de la communication comme quelque chose de sacré. La France n'est pas habituée à la communication horizontale. Le QG de la communication, c'est les USA. Ils ont démarré leur société avec la communication : la Common Law vient du bas. Nous la communication vient du haut: elle tombe. Le problème reste un dosage entre le devoir d'informer et la prise en considération des citoyens contre la communication promotionnelle intéressée. Il y a des sociétés de service public comme la SNCF, qui ont forcément une communication promotionnelle car elles rentrent dans un marché concurrentiel. Mais, elles y vont très prudemment. La privatisation d'EDF est basée uniquement sur les valeurs du service public. Une entreprise qui n'a rien compris, c'est France Télécom. Elle a sacrifiée les valeurs montantes à son portefeuille d'actions. Il y a un problème entre la prise en considération des hommes et de la communication promotionnelle. La SNCF fait de la communication promotionnelle sans oublier le service public. France télécom se trompe aujourd'hui, car il oublie les valeurs qui fondent son identité. On passe sous silence l'identité de l'entreprise. L'identité est fondamentale en communication, c'est le vécu interne dans l'institution publique. Si vous faites des images qui sont très différentes de l'identité de l'entreprise, vous faites craquer l'entreprise. Les valeurs montantes sont l'identité d'une marque. L'image d'un territoire ou d'une entreprise doit être en harmonie avec ses valeurs et son histoire. Avoir une image qui respecte l'identité est un gage d'une communication authentique. 